\title{
SEM Studies in Textile Finishing Processes
}

W. R. Goynes, D. V. Parikh, V. Edwards, and T. Vigo

Southern Regional Research Center, ARS, USDA, New Orleans, LA

Processing of textiles often requires special chemical finishing or mechanical treatments to provide products with increased consumer value. These processes are applied to woven, knitted, or nonwoven materials, but the changes required for successful treatments occur with individual fibers. Microscopic examinations of the finished materials can provide information to help understand how specific finishes work, to aid in further treatment development, or to trouble-shoot for inefficient processes. Three applications are discussed.

Nonwoven textile-products are a rapidly developing market. Early nonwovens were generally developed using synthetic fibers. While cotton fibers can contribute desirable properties of softness and absorbency to these products, some current methods for producing nonwovens depend on fiber fusibility and require fiber lengths that can only be provided by synthetic or high quality cotton fibers. Because most nonwoven materials are intended for markets of low-cost products, use of premium cotton fibers should be avoided where possible. Devising ways to incorporate low-grade cotton fibers into blended cotton/synthetic products is a challenge. Use of fusible synthetic fibers is one means of doing this. Webs of fused synthetic fibers can be used to form networks that entrap the linter bundles to prevent movement. The added body provided to the material by the cotton produces nonwoven sheets usable as padding or fillers. In development of these processes it is essential to understand the distribution of the fiber types within the product, and the nature and amount of fused bonding that has occurred. Figure 1A shows a nonwoven product using bicomponent, fusible polyester, and cotton fibers. Figure 1B shows distribution of these blended fibers and areas where fused bonds have formed.

Wound dressings that support healing and are body site conforming were prepared by modifying cotton gauzes to incorporate alginates. Cotton products provide good wound dressings but tend to dry into the wound. Alginate dressings require a cover dressing. Experimental combinations of these materials incorporate properties of both. The citrate conjugate of alginate and cellulose in these modified gauzes, formed through citric acid crosslinking of the cellulose and alginate, gels upon hydration. This provides a wound dressing that has conformabilty and elasticity ye maintains moisture at the wound site. Performance of the dressing for conserving moisture depends at least in part on the amount of alginate attached to the gauze, and the state to which it hydrates. Figure $2 \mathrm{~A}$ shows the gauze structure with the attached dry alginate. Figure 2B shows the changes in the appearance of the gauzealginate matrix on hydration.

Antibacterial agents were applied to cotton fabrics and blends of cotton with rayon, and polyester. These chemicals are externally bound on the fabric surfaces, and effectiveness and durability of the finishes depends on the amount of chemical bound to the surface and whether it remains attached after repeated launderings. SEM examinations showed that cotton had more bound agent before and after laundering than other fibers. This appeared to be due to undulated, non-spherical cotton fiber structures as compared to smooth, rounded surfaces of the synthetic fibers used. Heavy chemical deposits on a cotton fiber are shown in Figure 3A. Figure 3B shows remaining deposited chemicals after laundering. Deposits on smooth round fibers are more easily removed during laundering. 


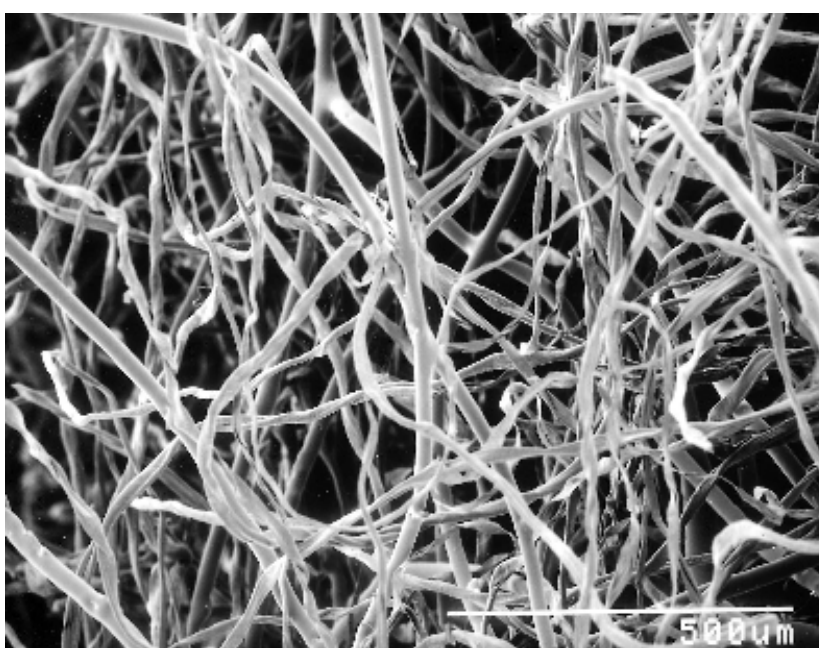

Figure 1A. Nonwoven of bicomponent fusible polyester and cotton

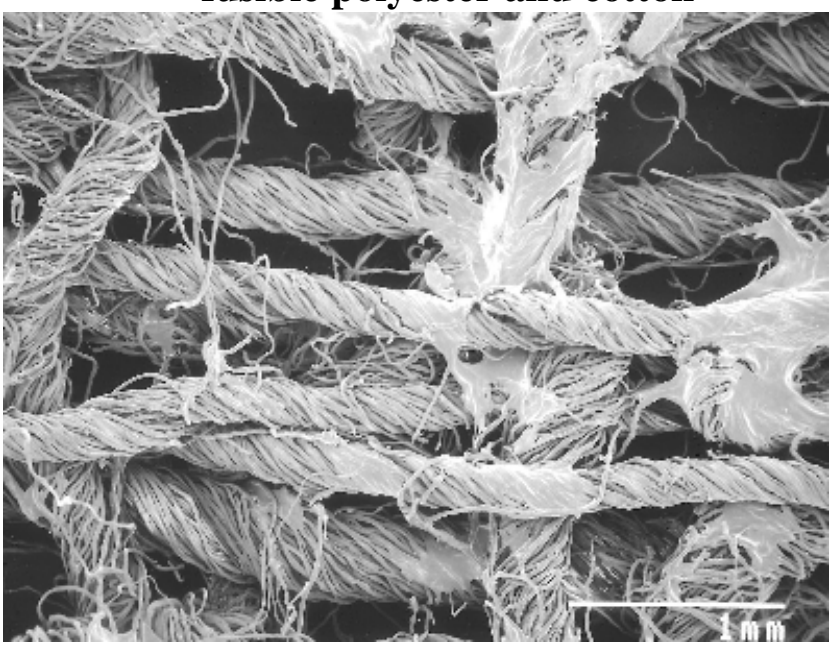

Figure 2A. Cotton gauze with attached dry alginate

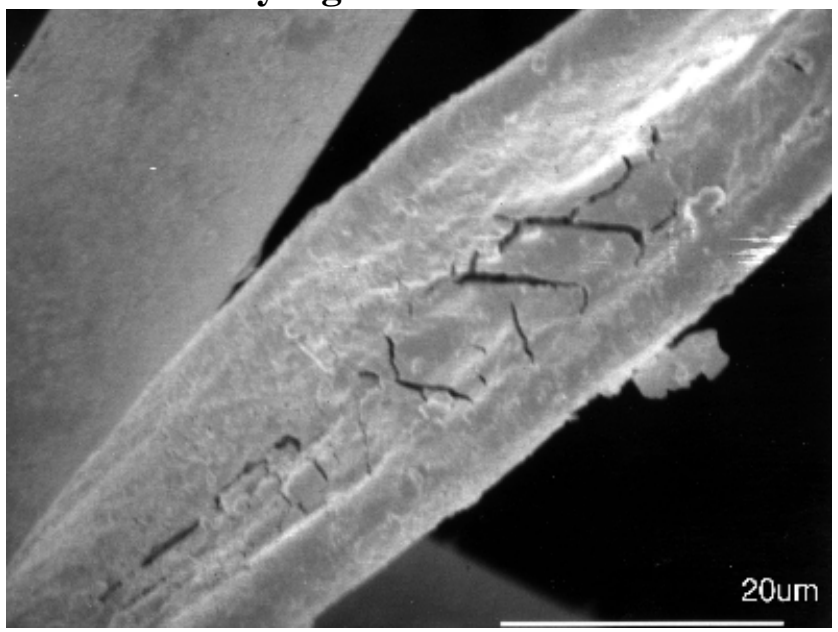

Figure 3A. Heavy chemical deposits on cotton fiber

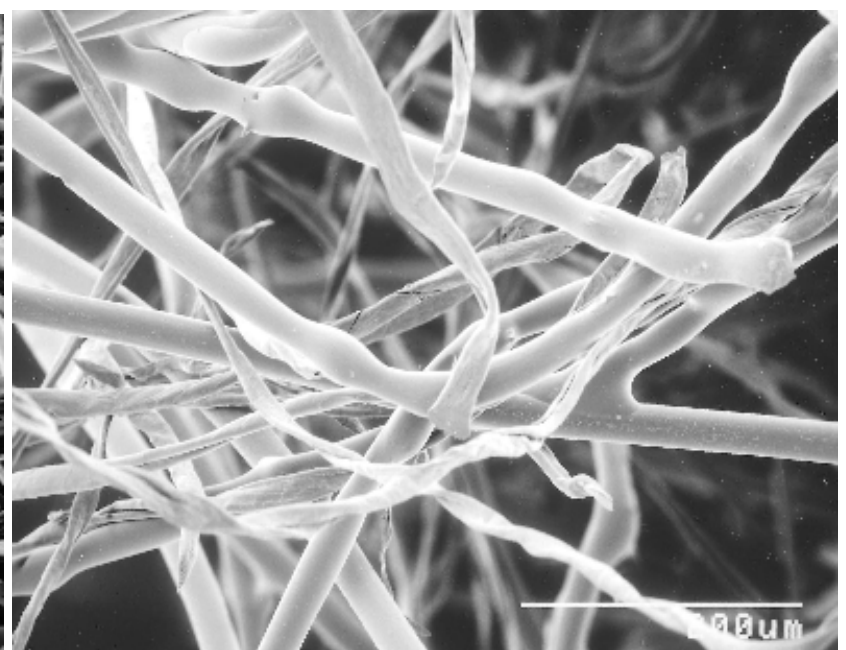

Figure1B. Fiber distribution and fused bonds in nonwoven

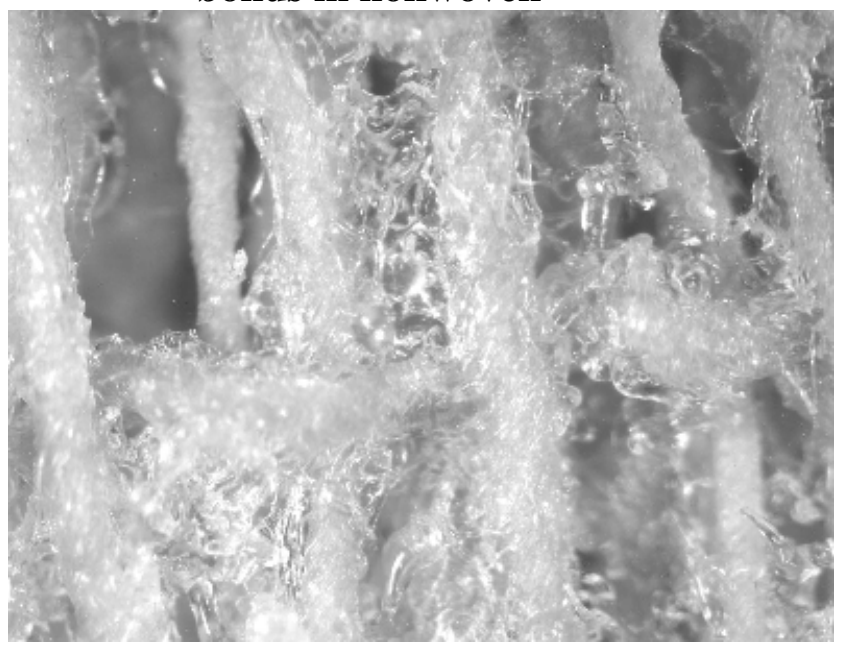

Figure 2B. Changes in appearance of gauze matrix on hydration

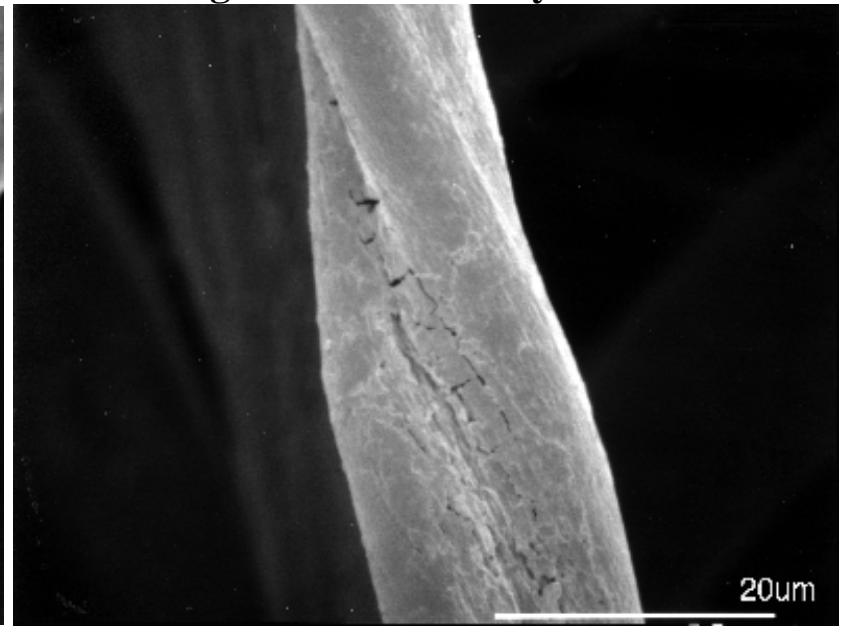

Figure 3B. Chemical deposits remaining on fiber after laundering 